\title{
A Thermostatted Kinetic Framework with Particle Refuge for the Modeling of Tumors Hiding
}

\author{
Carlo Bianca ${ }^{1, *}$, Christian Dogbe $e^{2}$ and Luca Guerrini ${ }^{3}$ \\ ${ }^{1}$ Dipartimento di Scienze Matematiche, Politecnico, Corso Duca Degli Abruzzi 24, 10129 Torino, Italy \\ 2 Department of Mathematics, Université de Caen, LMNO, CNRS, UMR 6139, 14032 Caen Cedex, France \\ ${ }^{3}$ Department of Management, Polytechnic University of Marche Ancona 60121, Italy
}

Received: 7 Mar. 2013, Revised: 8 Jul. 2013, Accepted: 10 Jul. 2013

Published online: 1 Mar. 2014

\begin{abstract}
Recently, thermostatted kinetic models have been proposed for the modeling of complex systems subjected to external force fields. This paper deals with the derivation of a generalized thermostatted kinetic framework, which incorporates particles refuge. From the qualitative viewpoint, the global in time existence and uniqueness of the solution to the relative Cauchy problem, the time evolution of the moments of the solution, and the existence of stationary solutions are discussed. Applications refer to biological systems and especially to tumor-escape from immune system surveillance, indeed within this framework an mathematical model for the tumorimmune system competition is derived. Further research directions are outlined in the last section of the paper.
\end{abstract}

Keywords: Populations dynamics, Thermostats, Active particles, Integro-differential equation, Tumor-escape

\section{Introduction}

The development of mathematical frameworks, possibly a mathematical theory, for the modeling of complex nonequilibrium living systems is a new challenge of the mathematicians of this century. Population dynamics models [1] for predator-prey interactions [2] have been extensively studied and have allowed to model systems at equilibrium [3]. Recently the thermostatted kinetic theory for active particles framework $[4,5,6,7,8]$ has been proposed for the modeling of nonequilibrium systems, namely systems subjected to external actions, see also [9].

In the context of predator-prey frameworks, the dynamics and bifurcations analysis of mathematical models with refuge have gained much attention, see, among others, papers $[10,11,12,13,14,15,16]$ and the review paper [17]. The use of refuge can be broadly defined to include any strategy that decreases predation risk, e.g., spatial or temporal refuges, prey aggregations, or reduced search activity by prey. The existence of refuges can clearly have important effects on the coexistence of predators and prey and on the stability of equilibrium solutions.

It is worth mentioning that the ways by which the effects of prey refuges have been incorporated in predator-prey interactions is to consider two types of refuges in the literature: those that protect a constant fraction of prey and those that protect a constant number of prey.

This paper aims at offering a further generalization of the thermostatted framework proposed in [21]. Specifically the thermostatted kinetic framework with conservative interactions is further explored and generalized in order to include the role of particles refuge in the time evolution equations of the distribution function. The distribution function includes a scalar variable (activity) for the modeling of the strategy performed by active particles. Applications of the thermostatted framework proposed in the present paper refer to the modeling of tumor-immune system competition and specifically to the ability of tumor cells to escape from immune system cells surveillance. This is an important issue in tumor growth. Indeed, it is well-know, that the immune system is able to protect the host from virus-induced tumors by eliminating or suppressing viral infection. Moreover the timely elimination of pathogens and prompt resolution of inflammation can prevent the establishment of an inflammatory environment conducive to tumorigenesis. Finally, the immune system can specifically identify and

\footnotetext{
*Corresponding author e-mail: carlo.bianca@ polito.it
} 
eliminate tumor cells on the basis of their expression of tumor-specific antigens or molecules induced by cellular stress (tumor immune surveillance), see the review paper [18]. However, if the immune response still fails to completely eliminate the tumor, the process results in the selection of tumor cell variants that are able to resist, avoid, or suppress the antitumor immune response, leading to the escape phase. During the escape phase, the immune system is no longer able to contain tumor growth, and a progressively growing tumor results, see, among others, papers $[19,20]$ and the references cited therein. The goal of this paper is the derivation of a mathematical framework for the modeling of this process.

The present paper is divided into more four sections, which follow this introduction. Specifically Section 2 introduces the generalized thermostatted kinetic framework with particles refuge for the modeling of complex systems subjected to external force fields. The existence and uniqueness of the solution for the mathematical framework proposed in Section 2, the evolution equation for the moments and the existence of stationary solutions are discussed in Section 3. A prototype model for the tumor escape from immune system surveillance during their competition is derived in Section 4. Finally concluding remarks and future research directions are outlined in Section 5.

\section{The Thermostatted Kinetic Framework with Particles Refuge}

This section is concerned with the derivation of the kinetic framework coupled with the Gaussian isokinetic thermostat with applications to the modeling of complex systems subjected to external force fields and such that some particles are able to refuge. Specifically we assume that the system is constituted by $n \in \mathbb{N}$ subsystems composed by active particles whose microscopic state includes the activity variable $u \in D_{u}$ (functional subsystems). The evolution of each functional subsystem is described by a probability distribution function $f_{i}=f_{i}(t, u):\left[0, \infty\left[\times D_{u} \rightarrow \mathbb{R}^{+}\right.\right.$, for $i \in\{1,2, \ldots, n\}$, such that the $p$ th order moment of the whole system reads:

$$
\mathbb{E}_{p}[\mathbf{f}](t)=\int_{D_{u}} u^{p} \tilde{f}(t, u)<\infty, \quad p \in \mathbb{N}
$$

where $\mathbf{f}=\mathbf{f}(t, u)=\left(f_{1}(t, u), f_{2}(t, u), \ldots, f_{n}(t, u)\right)$ and

$$
\widetilde{f}(t, u)=\sum_{i=1}^{n} f_{i}(t, u) d u .
$$

In particular $\mathbb{E}_{0}[\mathbf{f}]$ represents the particles density of the system and $\mathbb{E}_{2}[\mathbf{f}]$ the activation energy. Moreover the $p$ th order moment of each functional subsystem $f_{i}$ reads:

$$
\mathbb{E}_{p}\left[f_{i}\right](t)=\int_{D_{u}} u^{p} f_{i}(t, u)<\infty, \quad p \in \mathbb{N}
$$

In what follows we assume that some particles of a functional subsystem are able to refuge during the interactions. We model this behavior by assuming that no refuging particles have microscopic state $u \in R_{u} \subset D_{u}$. In particular, for explanation convenience, we assume that particles of the subsystem with distribution function $f_{1}(t, u)$ escape the interaction. Then it is not restrictive to assume that:

$$
f_{1}(t, u)=f_{1}(t, u) \chi_{R_{u}}(u)
$$

where $\chi_{R_{u}}$ is the characteristic (indicator) function of $R_{u}$. Therefore the $p$ th order moment of the whole system can be rewritten as follows:

$$
\mathbb{E}_{p}[\mathbf{f}](t)=\int_{R_{u}} u^{p} f_{1}(t, u) d u+\int_{D_{u}} u^{p} \widehat{f}(t, u) d u,
$$

where

$$
\widehat{f}(t, u)=\sum_{i=2}^{n} f_{i}(t, u) d u .
$$

We now derive the time evolution equation of each distribution $f_{i}$. In particular we assume that only conservative interactions occur, namely interactions do not modify the number of particles. Moreover mutual interactions refer to test particle, with distribution function $f_{i}(t, u)$, candidate particles (with distribution function $f_{i}\left(t, u_{*}\right)$ ) and field particles (with distribution function $\left.f_{i}\left(t, u^{*}\right)\right)$. Candidate particles can acquire the microscopic state of the test particle after interactions with field particles.

The possibility of interactions among the particles is measured by the nonnegative function $\eta_{i j}\left(u_{*}, u^{*}\right)$, which represents the interaction rate between the subsystem $f_{i}$ and the subsystem $f_{j}$; the probability that after this interaction the candidate particle undergoes a change in its microscopic state (that of test particle) is measured by the nonnegative function $\mathcal{A}_{i j}\left(u_{*}, u^{*}, u\right)$, which is a probability density with respect to $u$ and then

$$
\int_{D_{u}} \mathcal{A}_{i j}\left(u_{*}, u^{*}, u\right) d u=1, \quad \forall u_{*}, u^{*} \in D_{u} .
$$

Setting

$$
\Gamma_{i j}=\Gamma_{i j}\left(u_{*}, u^{*}, u\right)=\eta_{i j}\left(u_{*}, u^{*}\right) \mathcal{A}_{i j}\left(u_{*}, u^{*}, u\right),
$$

bearing all above in mind and summing up with respect to the all candidate and field particles we obtain the following operator which models the gain of test cells:

$$
\mathcal{G}_{i}[\mathbf{f}](t, u)= \begin{cases}\widetilde{\mathcal{G}}_{1}[\mathbf{f}](t, u), & i=1 \\ \widetilde{\mathcal{G}}_{i}[\mathbf{f}](t, u), & i \neq 1\end{cases}
$$

where

$$
\begin{aligned}
\widetilde{\mathcal{G}}_{1}[\mathbf{f}] & =\int_{R_{u} \times R_{u}} \Gamma_{11} f_{1}\left(t, u_{*}\right) f_{1}\left(t, u^{*}\right) d u_{*} d u^{*} \\
& +\sum_{j=2}^{n} \int_{R_{u} \times D_{u}} \Gamma_{1 j} f_{1}\left(t, u_{*}\right) f_{j}\left(t, u^{*}\right) d u_{*} d u^{*}
\end{aligned}
$$


and

$$
\begin{aligned}
\widetilde{\mathcal{G}}_{i}[\mathbf{f}] & =\int_{D_{u} \times R_{u}} \Gamma_{i 1} f_{i}\left(t, u_{*}\right) f_{1}\left(t, u^{*}\right) d u_{*} d u^{*} \\
& +\sum_{j=2}^{n} \int_{D_{u} \times D_{u}} \Gamma_{i j} f_{i}\left(t, u_{*}\right) f_{j}\left(t, u^{*}\right) d u_{*} d u^{*} .
\end{aligned}
$$

Similarly the lost of test cells is modeled by the following operator:

$$
\begin{aligned}
\mathcal{L}_{i}[\mathbf{f}] & =f_{i}(t, u) \int_{R_{u}} \eta_{i 1}\left(u, u^{*}\right) f_{1}\left(t, u^{*}\right) d u^{*} \\
& +f_{i}(t, u) \sum_{j=2}^{n} \int_{D_{u}} \eta_{i j}\left(u, u^{*}\right) f_{j}\left(t, u^{*}\right) d u^{*} .
\end{aligned}
$$

Assuming nonequilibrium conditions, namely the presence of external fields $F_{i}=F_{i}(u): D_{u} \rightarrow \mathbb{R}^{+}$at macroscopic scale, the thermostatted kinetic framework with particles refuge reads:

$$
\partial_{t} f_{i}+\partial_{u}\left(F_{i} f_{i}\right)-\mathcal{T}_{i}\left[F_{i}, \mathbf{f}\right]=\mathcal{C}_{i}[\mathbf{f}]=\mathcal{G}_{i}[\mathbf{f}]-\mathcal{L}_{i}[\mathbf{f}],
$$

where $\mathcal{T}_{i}\left[F_{i}, \mathbf{f}\right]=\mathcal{T}_{i}\left[F_{i}, \mathbf{f}\right](t, u)$ is the thermostatted term which reads:

$$
\mathcal{T}_{i}\left[F_{i}, \mathbf{f}\right]=\partial_{u}\left(u F_{i}\left(\int_{R_{u}} u f_{1} d u+\int_{D_{u}} u \widehat{f} d u\right) f_{i}\right) .
$$

The thermostatted term is a damping operator that is adjusted so as to control the activation energy.

Remark 1. It is easy to show that the term $\mathcal{C}_{i}[\mathbf{f}]$ is conservative, namely:

$$
\int_{D_{u}} \mathcal{C}_{i}[\mathbf{f}](t, u) d u=1
$$

for $i \in\{1,2, \ldots, n\}$.

It is worth stressing that particles refuge defined in the present paper can be also introduced in the hybrid frameworks developed and analyzed in the paper [6] and in the $p$-thermostatted framework proposed in the paper [7].

\section{On the Existence of Solutions}

This section is meant to analytical results on the mathematical framework (13). In particular we define the density of the non-refusing particles of the functional subsystem $f_{1}$ as follows:

$$
\mathbb{R}\left[f_{1}\right](t)=\int_{R_{u}} f_{1}(t, u) d t
$$

In what follows we assume that $F_{i}=F$ and $\eta_{i j}=\eta$ are real number and $\mathcal{A}_{i j}$ is an even function with respect to $u$. Moreover we assume that $\mathbb{E}_{0}[\mathbf{f}](t)=1$, and $f_{i}(t, u)=0$ as $(t, u) \in\left[0, \infty\left[\times \partial D_{u}\right.\right.$, for $i \in\{1,2, \ldots, n\}$.
Let $\mathcal{C}_{i}[\mathbf{f}]=\mathcal{G}_{i}[\mathbf{f}]-\mathcal{L}_{i}[\mathbf{f}]$ be the conservative operator; multiplying both sides of it by $u$ and integrating over $D_{u}$, we have:

$$
\int_{D_{u}} u \mathcal{C}_{i}[\mathbf{f}](t, u)=-\eta \mathbb{E}_{1}[\mathbf{f}]
$$

Multiplying the left hand side of (13) by $u$ and performing integration by parts on the second term, we have:

$$
\int_{D_{u}}\left(\partial_{u}\left(F_{i} f_{i}\right)-\mathcal{T}_{i}\left[F_{i}, \mathbf{f}\right]\right) d u=\left(\mathbb{E}_{1}[\mathbf{f}]\right)^{2}-1 .
$$

Therefore $\mathbb{E}_{1}[\mathbf{f}]$ is solution of the following differential equation:

$$
\frac{d}{d t} \mathbb{E}_{1}[\mathbf{f}]+F\left(\mathbb{E}_{1}[\mathbf{f}]\right)^{2}+\eta \mathbb{E}_{1}[\mathbf{f}]=F .
$$

The above equation admits a unique solution when an initial condition is assigned. Therefore we are able to define the mild solution of the relative abstract Cauchy problem for the thermostatted kinetic framework with refuge (13) and to perform the mathematical analysis developed in [21] regarding the existence and uniqueness of the solution. In particular the following relation holds true:

$$
\mathbb{E}_{0}[\mathbf{f}]=\int_{R_{u}} f_{1}(t, u) d u+\int_{D_{u}} \widehat{f}(t, u) d u=1 .
$$

Moreover we are able to prove the existence of stationary solutions by using measure theory and fixed point arguments, see [22].

\section{An Abstract Model for Tumor-Escape from Immune System}

The thermostatted framework with particles refuge (13) acts as paradigms for the derivation of models. The derivation of a specific model means providing, by a suitable phenomenological interpretation of the system, a mathematical description of the microscopic interactions among the selected functional subsystems.

This section is devoted to the derivation of a mathematical model for the tumor-escape from immune system. Therefore we consider two functional subsystems: tumor cells and immune system cells. Let $f_{1}(t, u)$ be the distribution function of the tumor cells, with $u \in R_{u}=[0,1]$, and $f_{1}(t, u)$ the distribution function of the immune system cells, with $u \in D_{u}=[-1,1]$. The activity variable $u$ represents tumor progression for tumor cells subsystem and activation for the immune system cells subsystem. Moreover we assume that there are not external constant force fields, namely $F_{i}(u)=0$. Finally we assume that the interaction rates $\eta_{11}, \eta_{12}, \eta_{21}, \eta_{22}$ are constant nonnegative numbers, and the probability density $\mathcal{A}_{i j}$ is assumed to be defined by a delta Dirac function 
(deterministic output $m_{i j}\left(u_{*}, u^{*}\right)$ of a pair interaction) depending on the microscopic state of the interacting pairs:

$$
\mathcal{A}_{i j}\left(u_{*}, u^{*}, u\right)=\delta\left(u-m_{i j}\left(u_{*}, u^{*}\right)\right) \text {. }
$$

The conservative term $C_{1}[\mathbf{f}](t, u)$, is derived under the assumption that tumor cells have a tendency to increase their microscopic state with a certain rate, regulated by the interactions with the immune system cells. $C_{2}[\mathbf{f}](t, u)=0$ is derived under the assumption that the activation of the immune system depends on the interactions with tumor cells. Accordingly, we define:

$$
m_{i j}\left(u_{*}, u^{*}\right)= \begin{cases}u_{*}+\varepsilon \alpha & \text { if } j=2 \text { and } i=1 \\ u_{*}+\alpha & \text { if } j=1 \text { and } i=2 \\ u_{*} & \text { otherwise }\end{cases}
$$

where $\alpha$ is a positive parameter, which defines the rate toward high states of activity, while $\varepsilon<1$ is a scale parameter. Straightforward calculations give the following conservative terms:

$C_{i}[\mathbf{f}]= \begin{cases}\eta_{12} \mathbb{E}_{0}\left[f_{2}\right]\left[f_{1}(t, u-\epsilon \alpha)-f_{1}(t, u)\right] & \text { if } i=1, \\ \eta_{21} \mathbb{R}\left[f_{1}\right]\left[f_{2}(t, u-\alpha)-f_{2}(t, u)\right] & \text { if } i=2 .\end{cases}$

Therefore the abstract kinetic mathematical model with particle refuge for tumor-escape from immune system surveillance reads:

$$
\left\{\begin{array}{l}
\partial_{t} f_{1}=\eta_{12}\left[f_{1}(t, u-\epsilon \alpha)-f_{1}(t, u)\right] \mathbb{E}_{0}\left[f_{2}\right] \\
\partial_{t} f_{2}=\eta_{21}\left[f_{2}(t, u-\alpha)-f_{2}(t, u)\right] \mathbb{R}\left[f_{1}\right]
\end{array}\right.
$$

where

$$
\mathbb{R}\left[f_{1}\right]=\int_{0}^{1} f_{1}(t, u) d u, \quad \mathbb{E}_{0}\left[f_{2}\right]=\int_{-1}^{1} f_{2}(t, u) d u .
$$

According to the above model, the immune system interacts with the tumor cells that are not escaped.

\section{Conclusions and Research Perspectives}

In this paper we have derived a thermostatted kinetic framework that can be proposed for the modeling of biological systems, especially in tumor-immune system competition, with particles refuge. Future research directions are outlined in this section.

The mathematical framework of this paper does not include the role of non conservative interactions (proliferative, destructive, mutative). The role of proliferative and/or destructive interactions are modeled by the following operator:

$\mathcal{N}_{i}[\mathbf{f}]=f_{i}(t, u) \sum_{j=1}^{n} \int_{\left(D_{u}\right)^{2}} \alpha_{i j}\left(u_{*}, u^{*}\right) f_{j}\left(t, u^{*}\right) d u_{*} d u^{*}$ where

$$
\alpha_{i j}\left(u_{*}, u^{*}\right)=\eta_{i j}\left(u_{*}, u^{*}\right) \mu_{i j}\left(u_{*}, u^{*}\right),
$$

being $\alpha_{i j}\left(u_{*}, u^{*}\right)$ the net proliferation rate.

In particular mutative interactions have an important role in tumor-immune system competition, see Nowak [23]. Therefore the thermostatted framework with particles refuge proposed in the present paper can be further generalized in order to take into account the role of mutations, which are modeled by the following operator:

$$
\mathcal{M}_{i}[\mathbf{f}]=\sum_{h=1}^{n} \sum_{k=1}^{n} \int_{\left(D_{u}\right)^{2}} \beta_{h k}^{i} f_{h}\left(t, u_{*}\right) f_{k}\left(t, u^{*}\right) d u_{*} d u^{*},
$$

where

$$
\beta_{h k}^{i}=\beta_{h k}^{i}\left(u_{*}, u^{*}, u\right)=\eta_{h k}\left(u_{*}, u^{*}\right) \varphi_{h k}^{i}\left(u_{*}, u^{*}, u\right),
$$

being $\varphi_{h k}^{i}\left(u_{*}, u^{*}, u\right)$ the net proliferation rate into the $i$ th functional subsystem, due to interactions that occur with rate $\eta_{h k}$, of the candidate $h$-particle, with state $u_{*}$, and the field $k$-particle, with state $u^{*}$.

It is worth stressing that nonconservative interactions may not ensure the global existence of solutions.

A future research perspective is the derivation of formal asymptotic limits for the derivation of macroscopic equations by employing the mathematical methods developed in papers [24,25,26] that use parabolic and/or hyperbolic scaling.

Finally we would like to underline that the introduction of particle refuge can be also performed in the interaction rates. This is a work in progress and results will be presented in due course.

\section{Acknowledgement}

The first author acknowledges the financial support by the FIRB project-RBID08PP3J-Metodi matematici e relativi strumenti per la modellizzazione e la simulazione della formazione di tumori, competizione con il sistema immunitario, e conseguenti suggerimenti terapeutici.

\section{References}

[1] P. Turchin, Complex Population Dynamics: A Theoretical/Empirical Synthesis, Princeton University Press, (2003).

[2] P. A. Abrams, Annu. Rev. Ecol. Syst, 31, 79-105 (2000).

[3] C. J Briggs, M. F. Hoopes, Theoretical Population Biology, 65, 299-315 (2004).

[4] C. Bianca, Applied Mathematical Sciences, 6, 651-660 (2012).

[5] C. Bianca, Int. Journal of Math. Analysis, 6, 813-824 (2012).

[6] C. Bianca, Nonlinear Analysis: Real World Applications, 13, 2593-2608 (2012). 
[7] C. Bianca, M. Ferrara, L. Guerrini, Journal of Global Optimization, doi:10.1007/s10898-013-0045-2, (2013).

[8] C. Bianca, The Scientific World Journal, doi:http://dx.doi.org /10.1155/2013/274719, (2013).

[9] C. Bianca, Physics of Life Reviews, 9, 359-399 (2012).

[10] A. Sih, Theor. Pop. Biol., 31, 1-12 (1987).

[11] H. Ylönen, R. Pech, S. Davis, Evol. Ecol., 17, 349-369 (2003).

[12] T. K. Kar, Communications in Nonlinear Science and Numerical Simulation, 10, 681-691 (2005).

[13] V. Krivan, J Theor Biol., 274, 67-73 (2011).

[14] F. Chen, Z. Ma, H. Zhang, Nonlinear Analysis: Real World Applications, 13, 2790-2793 (2012).

[15] X. Guan, W. Wang, Y. Cai, Nonlinear Analysis: Real World Applications, 12, 2385-2395 (2011).

[16] S. Wang, Z. Ge, Abstract and Applied Analysis, doi:http://dx.doi.org/10.1155/2013/168340, (2013).

[17] Z. Maa, W. Li, Y. Zhao, W. Wangb, H. Zhang, Z. Li, Mathematical Biosciences, 218, 73-79 (2009).

[18] J. B. Swann and M. J. Smyth, J Clin Invest, 117, 1137-1146 (2007).

[19] R. T.Costello, J. A. Gastaut, D. Olive, Arch Immunol Ther Exp (Warsz), 47, 83-88 (1999).

[20] A. P. Vicari, C. Caux, G. Trinchieri, Semin Cancer Biol, 12, 33-42 (2002).

[21] C. Bianca, Appl. Math. Inf. Sci., 6, 495-499 (2012).

[22] C. Bianca, Mathematical Methods in the Applied Sciences, 36, 1768-1775 (2013).

[23] M. Nowak, Evolutionary Dynamics: Exploring the Equations of Life, Belknap Press, (2006).

[24] C. Bianca and C. Dogbe, Nonlinear Studies, 20, 281-305 (2013).

[25] A. Bellouquid and C. Bianca, Math. Comput. Modelling, 52, 802-813 (2010).

[26] P. Degond, B. Wennberg, Commun. Math. Sci., 5, 355-382 (2007).

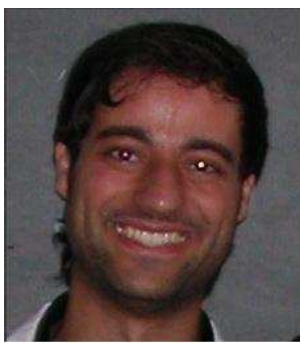

Carlo Bianca received the $\mathrm{PhD}$ degree in Mathematics for Engineering Science at Polytechnic University of Turin. His research interests are in the areas of applied mathematics and in particular in mathematical physics including the mathematical methods and models for complex systems, mathematical billiards, chaos, anomalous transport in microporous media and numerical methods for kinetic equations. He has published research articles in reputed international journals of mathematical and engineering sciences. He is referee and editor of mathematical journals.

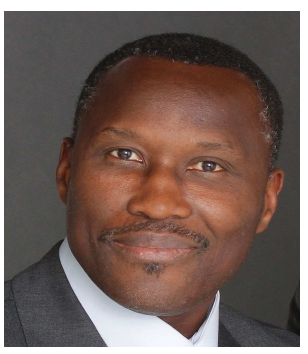

Christian Dogbe received the $\mathrm{PhD}$ degree in Applied Mathematics at University Denis Diderot Paris 7 of Paris. His research interests are in the areas of applied mathematics and in particular in mathematical physics, including the mathematical methods and models for complex systems, mathematical analysis, Boltzmann equation and numerical methods for kinetic equations, computer science, mechanical engineering. He has published research articles in reputed international journals of mathematical and engineering sciences.

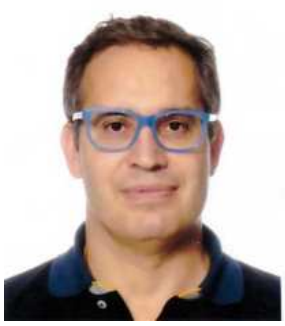

Luca Guerrini is Associate Professor of Mathematical Economics at the Polytechnic University of Marche, Italy. He holds an MA and $\mathrm{PhD}$ in Pure Mathematics from the University of California, Los Angeles, USA. His research interests are in the areas of pure and applied mathematics as well as mathematical economics. He has published extensively in internationally refereed journals. 